\title{
Damping rates of p-modes by an ensemble of randomly distributed thin magnetic flux tubes
}

\author{
Andrew Gascoyne and Rekha Jain \\ Department of Applied Mathematics, University of Sheffield, UK \\ email: app07adg@sheffield.ac.uk, R.Jain@sheffield.ac.uk
}

\begin{abstract}
The magnetohydrodynamic (MHD) sausage tube waves are excited in the magnetic flux tubes by p-mode forcing. These tube waves thus carry energy away from the p-mode cavity which results in the deficit of incident p-mode energy. We calculate the loss of incident p-mode energy as a damping rate of $\mathrm{f}$ - and p-modes. We calculate the damping rates of $\mathrm{f}$ - and $\mathrm{p}$-modes by a model Sun consisting of an ensemble of many thin magnetic flux tubes with varying plasma properties and distributions. Each magnetic flux tube is modelled as axisymmetric, vertically oriented and untwisted. We find that the magnitude and the form of the damping rates are sensitive to the plasma- $\beta$ of the tubes and the upper boundary condition used.
\end{abstract}

Keywords. Sun: magnetic fields, Sun: helioseismology, Sun: oscillations, MHD, Scattering

\section{Introduction}

Solar acoustic modes (p-modes) can get absorbed and scattered when travelling through active regions such as sunspots or plages. This scattering of p-modes has been well observed using various helioseismic techniques (see Braun et al. 1988; Couvidat et al. 2006). Braun \& Birch (2008) found that sunspots can absorb over half of incident p-mode power and plage regions can absorb around $20 \%$. Also, it is found that the absorption is frequency dependent. Thus, the importance of sub-surface field structure in modifying the properties of $\mathrm{f}$ - and p-modes has been realised and many theoretical investigations have concentrated on understanding the physical mechanism responsible for this absorption (e.g., Spruit 1991; Bogdan \& Cally 1995; Bogdan et al. 1996; Crouch \& Cally 2005; Hindman \& Jain 2008; Jain et al. 2009).

In this paper we wish to study the combined effects of the upper boundary condition and the tube parameter plasma- $\beta$ on the damping rates of p-modes. We construct a model consistent with Hindman \& Jain (2008) in order to calculate damping rates produced by the excitation of sausage tube waves across the whole Sun. In Hindman \& Jain (2008) they used an idealised model where by each tube of the ensemble was identical; this need not be the case. Thus we use a random ensemble of thin, axisymmteric, vertical magnetic flux tubes with varying plasma- $\beta$.

\section{Equilibrium configuration}

We model the nonmagnetic medium as a polytropic atmosphere with gravity acting downwards $\mathbf{g}=-g \hat{\mathbf{z}} ; z$ is increasing upwards. The pressure, density and sound speed vary as power laws (see Bogdan et al. 1996 for details). Following Bogdan et al. (1996), Hindman \& Jain (2008) and Jain et al. (2009) we set the truncation depth at $z=-z_{0}$ 
which is our model photosphere where the quantities $\rho_{0}$ and $P_{0}$ are the values of the mass density and gas pressure at this depth. Above the truncation depth $z>-z_{0}$ we assume the existence of a hot vacuum $\left(\rho_{\text {ext }} \rightarrow 0\right.$ with temperature $\left.T_{\text {ext }} \rightarrow \infty\right)$. The external pressure, density and sound speed increases with depth and as our atmosphere is in convective equilibrium, the polytropic index $a$ is related to the ratio of specific heats $\gamma$ via $a=1 /(\gamma-1)$; which we set to $a=1.5$. We set the following characteristic physical parameters for our model photosphere, $\rho_{0}=2.78 \times 10^{-7} \mathrm{~g} \mathrm{~cm}^{-3}, P_{0}=1.21 \times 10^{5} \mathrm{~g} \mathrm{~cm}^{-1}$ $\mathrm{s}^{-1}$, and $g=2.775 \times 10^{4} \mathrm{~cm} \mathrm{~s}^{-1}$, which coincide with the photospheric reference model of Maltby et al. (1986). The choice of polytropic index $a=1.5$ yields the truncation depth $z_{0}=392 \mathrm{~km}$ and thus the sound speed at the surface is $8.52 \mathrm{~km} \mathrm{~s}^{-1}$.

We also assume that the magnetic fibrils threading the atmosphere are untwisted, straight, vertically aligned, thin tubes with a circular cross-section. For these thin magnetic flux tubes, the lateral variation of the magnetic field across the tube is ignored and the plasma $\beta$ is constant with depth (see Bogdan et al. 1996 and Hindman \& Jain 2008 for details).

\section{The governing wave equations}

The incident acoustic wavefield can be expressed as a single partial differential equation for the displacement potential $\Phi$,

$$
\frac{\partial^{2} \Phi}{\partial t^{2}}=c^{2} \nabla^{2} \Phi-g \frac{\partial \Phi}{\partial z}
$$

This equation supports plane wave solutions of the form,

$$
\Phi(\boldsymbol{x}, t)=\mathcal{A} e^{i(k x-\omega t)} Q(z), \quad Q(z)=w^{-(\mu+1 / 2)} W_{\kappa, \mu}(w),
$$

where $\mathcal{A}$ is the complex wave amplitude, $\omega$ the temporal frequency, and $k$ the wavenumber. $Q(z)$ is the vertical eigenfunction proportional to Whittaker's $W$ function (Abramowitz \& Stegun 1964), with $\mu=(a+1) / 2, \nu^{2}=a \omega^{2} z_{0} / g$ and $\kappa=\nu^{2} /\left(2 k z_{0}\right)$. We calculate the eigenvalues and eigenfunctions for the truncated polytrope by requiring that the Lagrangian pressure perturbation vanishes at the truncation depth, i.e., $\nabla \cdot \xi=0$. Mathematically this takes the form $W_{\kappa, \mu+1}\left(2 k z_{0}\right)=0$.

We are seeking waves on thin magnetic tubes that lack internal structure, so only three types of MHD waves can possibly satisfy this criterion: torsional Alfvén waves, longitudinal (sausage) waves and transverse (kink) waves. We ignore torsional Alfvén waves as our driving force, the p-mode oscillations, are irrotational in this model and therefore, will not generate these types of waves. In this paper we will also ignore the kink waves and specifically concentrate on the $m=0$ mode ( $m$ being the azimuthal order). Using the formulation of Jain et al. (2009) (see also Hindman \& Jain 2008 and Bogdan et al. 1996), the fluid displacement due to the excitation of sausage waves within the tube can be described by the following equation,

$$
\left(\frac{\partial^{2}}{\partial t^{2}}+\frac{2 g z}{2 a+\beta(1+a)} \frac{\partial^{2}}{\partial z^{2}}+\frac{g(1+a)}{2 a+\beta(1+a)} \frac{\partial}{\partial z}\right) \xi_{\|}=\frac{(1+a)(\beta+1)}{2 a+\beta(1+a)} \frac{\partial^{3} \Phi}{\partial z \partial t^{2}} .
$$

These sausage waves $\xi_{\|}(z, t)$ can be described as axisymmetric pressure pulses that produce displacements that are primarily parallel to the magnetic field. The p-mode driver 
term appears on the right hand side of equation (3.3) represented by the displacement potential $\Phi$.

\section{Damping rates for a random ensemble of tubes}

In order to calculate the damping rates $\Gamma$ for the excitation of sausage waves we follow Hindman \& Jain (2008). Thus we derive for a single flux tube, the energy flux of sausage tube waves that escape the p-mode cavity as

$$
E=-\frac{\gamma \beta}{2+\gamma \beta} \frac{\pi g \rho_{0} \omega A_{0}}{4(a+1)(\beta+1)} \frac{|\mathcal{A}|^{2}}{z_{0}^{2}}\left(\left|\Omega+\mathcal{I}^{*}\right|^{2}+|\mathcal{I}|^{2}-|\Omega|^{2}+\mathcal{S}\right) .
$$

Since we are interested in calculating the energy flux produced over the entire Sun, the energy flux produced by a single flux tube is not enough. Instead we need to calculate the total energy flux $E_{t o t}$ produced by an ensemble of flux tubes. Thus $E_{t o t}$ is simply the energy flux $E$ for a single tube multiplied by the number of tubes $N$ on the solar surface. The number of flux tubes depends only on the surface cross-sectional area of the tubes $A_{0}$ and the filling factor $f$, thus $N=4 \pi R_{\odot}^{2} f / A_{0}$, where $R_{\odot}$ is the radius of the Sun. We then define the damping rate for the excitation of sausage waves as,

$$
\begin{aligned}
\Gamma & =-\frac{1}{2 \pi} \frac{E_{t o t}}{E_{n}} \\
& =\frac{\beta}{4(\beta+1) \epsilon} \frac{\omega A_{0}}{4 \pi R_{\odot}^{2}} \frac{\lambda^{a}}{\nu^{4}} \frac{\left(\left|\Omega+\mathcal{I}^{*}\right|^{2}+|\mathcal{I}|^{2}-|\Omega|^{2}+\mathcal{S}\right)}{\mathcal{N}_{n}} N
\end{aligned}
$$

where $\lambda=2 k z_{0}, E_{n}=4 \pi R_{\odot}^{2} \frac{g \rho_{0} \nu^{4}|\mathcal{A}|^{2}}{4 a \lambda^{a} z_{0}^{2}} \mathcal{N}_{n}$ is the energy contained in f- and p-mode oscillations, $\left(\mathcal{N}_{n}\right.$ is an integral which can be found in Hindman \& Jain 2008 eqn. 3.14).

The next step is to simulate the aggregate behavior of the Sun by considering a random distribution of flux tubes for a range of $\beta$ values between 0 and 10 . We shall use three different random distributions, a uniform distribution so all $\beta$ values have equal probability. We shall also consider a normal (Gaussian) distribution of flux tubes and a exponential distribution such that the probability decreases as $\beta$ increases. We are given the number of tubes required by the parameter $N$, which requires us to keep the crosssectional $A_{0}$ of each tube the same. Thus $\beta$ has the effect of changing the magnetic flux $\Theta$ of the tube.

\section{Results and discussion}

We now present the results for theoretically calculated damping rates of p-modes by the generation of magnetic tube waves in the convection zone through external p-mode buffeting. We calculated damping rates for two different upper boundary conditions (BC), the stress-free condition which essentially means that all propagating tube waves are reflected at the surface and the maximal-flux condition which means all upward propagating tube waves escape into the atmosphere; this BC gives an upper bound on the amount of energy escaping the p-mode cavity.

We consider a random distribution of magnetic fibrils with $\beta$ values ranging from 0 to 10 as discussed in $\S 4$. We use three different random distributions, uniform, normal (Gaussian), and an exponential distribution and plot our results in figure 1. For comparison we have also over plotted the case $\beta=5$ with dashed lines for each mode order. For 

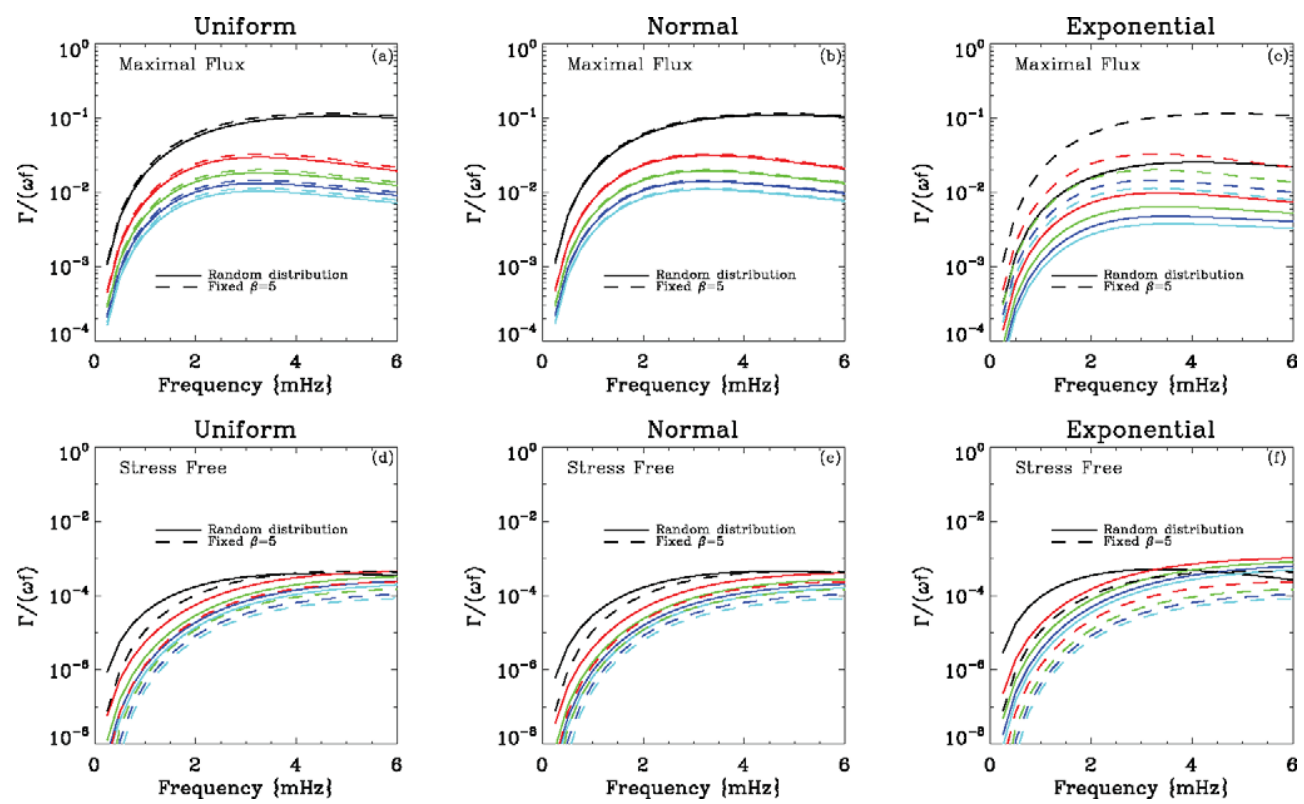

Figure 1. Damping rate of $\mathrm{f}$ - and p-modes calculated as a function of frequency for various random distributions in $\beta$, between $0-10$. Over-plotted with dashed lines, is the case for $\beta=5$. Panels (a)-(c) are for the maximal flux BC and panels (d)-(f) are for the stress free BC. Each mode order is denoted by a different colour: black (f), red (p1), green (p2), blue (p3) etc.

the maximal-flux case, the solid and dashed curves are similar for the uniform distribution and increasingly so for the normal distribution. For the exponential case the solid curve drops well below the dashed, this is due to the fact that this distribution dominates for lower $\beta$ values. For the stress free condition there is less correlation with the fixed $\beta=5$ case this is due to the fact that the damping rates depend nonlinearly on $\beta$.

\section{Conclusion}

In this paper we have presented a calculation for the damping rates for a random ensemble of vertical, axisymmetric, thin magnetic flux tubes. The mechanism used here is the generation of longitudinal (sausage) tube waves through buffeting of the magnetic fibril by external solar p-modes. The generation of these tube waves carry energy out of the p-mode cavity and thus result in the damping of p-modes. We have shown that there are a number of factors to consider when investigating the damping rates of p-modes by magnetic regions. In particular, the effect of the upper boundary condition and the distribution used to construct the random ensemble.

\section{References}

Abramowitz, M. \& Stegun, I. A. 1964, Handbook of Mathematical Functions (New York: Dover) Bogdan, T. J. \& Cally, P. S. 1995, Astrophys. J., 453, 919

Bogdan, T. J., Hindman, B. W., Cally, P. S., \& Charbonneau, P. 1996, Astrophys. J., 465, 406 Braun, D. C., Duvall, T. L., \& LaBonte, B. J. 1988, Astrophys. J., 335, 1015

Braun D. C., Birch, A. C. 2008, Solar Phys., 251, 267

Couvidat, S., Birch, A. C., \& Kosovichev, A. G. 2006, Astrophys. J., 640, 516 
Crouch, A. D. \& Cally, P. S. 2005, Solar Phys., 227, 1

Hindman, B. W. \& Jain, R. 2008, Astrophys. J., 677, 769

Jain R., Hindman, B., Braun, D. C., \& Birch, A. C. 2009, Astrophys. J., April 10 issue.

Maltby, P., Avrett, E. H., Carlsson, M., Kjelsdeth-Moe, O., Kurucz, R. L., \& Loeser, R. 1986, Astrophys. J., 306, 284

Spruit, H. C. 1991, in Toomre J., Gough D. O., eds, Lecture Notes in Physics, Vol. 388, Challenges to Theories of the Structure of Moderate Mass Stars. Springer-Verlag, Berlin, p. 121 